\title{
Targeted Intraoperative Radiotherapy Tumour Bed Boost during Breast-Conserving Surgery after Neoadjuvant Chemotherapy - a Subgroup Analysis of Hormone Receptor-Positive HER2-Negative Breast Cancer
}

\author{
Hans-Christian Kolberg ${ }^{a} \quad$ György Lövey ${ }^{b}$ Leyla Akpolat-Basci ${ }^{a} \quad$ Miltiades Stephanou $^{a}$ \\ Peter A. Fasching $^{c}$ Michael Untch ${ }^{d}$ Oliver Hoffmann ${ }^{e}$ Max Bulsara ${ }^{f, g}$ Jayant S. Vaidya ${ }^{g}$ \\ Cornelia Liedtke ${ }^{\mathrm{h}}$ \\ a Department of Gynaecology and Obstetrics, Marienhospital Bottrop, Bottrop, Germany; \\ ${ }^{b}$ Department of Radiation Oncology, BORAD, Bottrop, Germany; \\ ${ }^{\mathrm{c}}$ Department of Gynaecology and Obstetrics, University of Erlangen, Erlangen, Germany; \\ ${ }^{\mathrm{d} D e p a r t m e n t}$ of Gynaecology and Obstetrics, Helios Klinikum Berlin-Buch, Berlin, Germany; \\ e Department of Gynaecology and Obstetrics, University Hospital Essen, Essen, Germany; \\ ${ }^{f}$ Department of Biostatistics, University of Notre Dame, Fremantle WA, Australia; \\ g Department of Surgery and Interventional Science, University College London, London, UK; \\ h Department of Gynaecology and Obstetrics, University Hospital Schleswig-Holstein / Campus Lübeck, Lübeck, Germany
}

\section{Keywords}

Intraoperative radiotherapy - TARGIT-IORT .

Neoadjuvant therapy · Breast cancer · Breast-conserving surgery $\cdot$ Hormone receptor-positive $\cdot$ HER2-negative

\section{Summary}

Introduction: In a previous study our group showed a beneficial effect of targeted intraoperative radiotherapy (TARGIT-IORT) as an intraoperative boost on overall survival after neoadjuvant chemotherapy (NACT) compared to an external boost (EBRT). In this study we present the results of a detailed subgroup analysis of the hormone receptor (HR)-positive HER2-negative patients. Methods: In this cohort study involving 46 patients with HR-positive HER2-negative breast cancer after NACT, we compared the outcomes of 21 patients who received an IORT boost to those of 25 patients treated with an EBRT boost. All patients received whole breast radiotherapy. Results: Median follow-up was 49 months. Whereas disease-freesurvival and breast cancer-specific mortality were not significantly different between the groups, the 5-year Kaplan-Meier estimate of overall mortality was significantly lower by $21 \%$ with IORT, $p=0.028$. Non-breast cancer-specific mortality was significantly lower by $16 \%$ with IORT, $p=0.047$. Conclusion: Although our results have to be interpreted with caution, we have shown that the improved overall survival demonstrated previously could be reproduced in the HR-positive HER2-negative subgroup. These data give further support to the inclusion of such patients in the TARGIT-B (Boost) randomised trial that is testing whether IORT boost is superior to EBRT boost.

(c) 2017 S. Karger GmbH, Freiburg

\section{Introduction}

Partial irradiation of the breast with targeted intraoperative radiotherapy (TARGIT-IORT) using an intraoperative dose of 20 Gray (Gy) with a $50 \mathrm{kV}$ X-ray source is an increasingly used option for selected patients within a risk-adapted approach to replace whole breast irradiation after breast-conserving surgery for breast cancer [1]. Although the results of the TARGIT-A trial demonstrated a non-inferiority of this approach after a careful risk stratification [2], the concept of reducing the extent of radiation is not unopposed and remains an issue of constant debate [3].

\section{KARGER}

() 2017 S. Karger GmbH, Freiburg
Dr. med. Hans-Christian Kolberg 
The use of TARGIT-IORT as an intraoperative boost has been an option for much longer. The first studies using this technique as a replacement for the external boost demonstrated a reproducible local recurrence rate of $1.76 \%$ after 5 years rather than the expected $4.3 \%$ for the external boost $[4,5]$. Even when used in high-risk patient cohorts such as patients with triple-negative (TN) breast cancer in a trial using electrons as an intraoperative radiation (IOERT), the intraoperative boost resulted in a favourable outcome [6]. However, although the use of intraoperative radiotherapy as an intraoperative boost is widely used in primary surgery, its use in patients who undergo breast-conserving surgery after neoadjuvant chemotherapy (NACT) is not yet a standard.

NACT has become a standard of care not only for inoperable or locally advanced cases, but also for smaller operable tumours. Although NACT has been proven to increase the rate of breast-conserving surgeries, this is commonly not regarded as main rationale for its use. Instead, it is commonly regarded as an option for all patients where systemic therapy is definitely indicated at the time of diagnosis with the goal of improving disease-free survival (DFS) and overall survival (OS) [7]. NACT offers the potential for response-guided treatment through allowing an in vivo observation of chemotherapy sensitivity in an individual tumour. Regimens used in the neoadjuvant setting in clinical practice are usually the same as in adjuvant therapy.

Use of chemotherapy and especially NACT in HER2-positive and TN breast cancer is common clinical practice, but high-risk hormone receptor (HR)-positive HER2-negative patients with tumours showing a high proliferation rate or further risk factors such as grade 3 or high-risk classification based on a multigene assay may benefit from cytotoxic therapy and are, therefore, also potential candidates for NACT [8]. Achieving a pathological complete response ( $\mathrm{pCR}$ ) is considered indicative for a favourable prognosis [9]. These considerations have led to an increasing number of patients receiving neoadjuvant systemic therapy before breast surgery.

Patients receiving neoadjuvant systemic therapy have a higher risk of local and distant recurrence because they represent a cohort with an unfavourable tumour biology. Based on the hypothesis that these high-risk patients might benefit from the better disease control achieved by intraoperative radiotherapy as a boost as mentioned above, several groups have investigated this approach. Electrons as an intraoperative boost (IOERT) after primary systemic therapy were found to achieve excellent local control rates and a trend for superiority compared to a cohort receiving an external boost [10]. First data for the use of IORT with the $50 \mathrm{kV}$ X-ray source in this indication were presented by our study group in 2015 and showed a favourable outcome in a 1-arm observational design [11].

A previous study from our group of 116 patients showed a statistically beneficial effect of TARGIT-IORT after NACT on OS, but not DFS, compared to an external boost [12]. Although these data have to be interpreted with caution because of the retrospective design of the study and the small size of the cohorts, they may be inferred as a signal of non-inferiority of TARGIT-IORT as an intra- operative boost. An analysis of the subset of patients at highest risk with TN and HER2-positive tumours from this cohort showed a similar result for DFS but only a trend for a better OS without reaching statistical significance [13]. Based on these results we hypothesized that the effect, especially on OS, in the unselected cohort was driven by the HR-positive HER2-negative subgroup. The local recurrence rate after NACT shows significant differences according to tumour biology and in our rather short follow-up of 49 months a difference in local control for a subgroup representing luminal tumours was rather improbable [13]. Although the benefit for distant DFS in our unselected cohort differed significantly, the OS results were driven by non-breast-cancer mortality. Since the OS benefit could not be reproduced in the analysis of the subgroup of TN and HER2-positive patients with a high risk of breast cancer recurrence, we hypothesized that not only was the OS benefit clearly driven by the HR-positive HER2-negative subgroup, but also that the benefit on non-breast-cancer mortality could be demonstrated in these patients with a more favourable prognosis regarding breast cancer. Here we present the results for this subgroup.

\section{Patients and Methods}

\section{Patients}

TARGIT-IORT was introduced at the Marienhospital, Bottrop, Germany, in 2010 and from April 2010 all patients undergoing breast-conserving therapy after NACT were treated with a TARGIT-IORT boost (20 Gy to the surface of the applicator) during their surgery. The use of IORT as an intraoperative boost was performed according to the national guidelines in Germany. Patients were counselled with regards to the use of IORT by a radiooncologist and a breast surgeon in an interdisciplinary setting. All patients gave their informed consent. The local ethics committee approved retrospective analysis of the data.

We performed a longitudinal non-randomised retrospective cohort study based on data from the centre's database from 21 consecutive patients with HRpositive and HER2-negative tumours treated between April 2010 and November 2011 as the experimental TARGIT group. The control group consists of 25 consecutive patients treated with breast-conserving therapy after NACT in the previous 13 months (396 days). HR-positive tumours were defined as more than $0 \%$ stained cells in immunohistochemistry for oestrogen and progesterone receptor and HER2-negative either by 0 or $1+$ in immunohistochemistry or $2+$ and negative by fluorescence in-situ hybridisation. Postoperative external beam radiotherapy (EBRT) boost with 16 Gy in 8 fractions was given to all patients in the control EBRT group. All patients in both groups received whole breast radiotherapy with 50 Gy in 25 fractions. Radiotherapy to the supraclavicular lymph nodes was given to all patients with 4 or more histologically proven positive lymph nodes (positive either before or after NACT). Positive lymph nodes were confirmed with either a sentinel lymph node biopsy or core-cut biopsy performed before the start of NACT. Patients with positive nodes received an axillary dissection at surgery after NACT.

A titanium clip was placed in all tumours before NACT and the localisation of the original tumour bed prior to surgery was performed using a needle placed under monitoring by mammography (in the patients with a clinical complete remission) or sonography (in the patients with sonographically detectable residual disease). All cases underwent intraoperative radiography with identification of the titanium clip. In the patients of the control group with a planned external boost, 5 clips representing all levels of the tumour bed were placed intraoperatively.

pCR in this study was defined as no residual invasive or non-invasive tumour in breast or lymph nodes. All patients in this study had negative margins 
Table 1. Patient and tumour characteristics

\begin{tabular}{lccc}
\hline & IORT + & IORT- & $\mathrm{p}$ \\
\hline $\begin{array}{l}\text { Patients, } \mathrm{n} \\
\text { Tumour size, mm }\end{array}$ & 21 & 25 & \\
$\quad$ Mean & 26 & 27.84 & \\
SD & 11.63 & 9.50 & 0.558 \\
Grading & & & \\
G1 & 0 & 1 & $1^{\mathrm{a}}$ \\
G2 & 13 & 14 & \\
G3 & 8 & 10 & $1^{\mathrm{a}}$ \\
Nodal status & & & \\
positive & 14 & 16 & $1^{\mathrm{a}}$ \\
negative & 7 & 9 & \\
pCR & & 3 & \\
positive & 4 & 18 & \\
negative & 17 & & \\
\hline
\end{tabular}

${ }^{\text {aFishers exact. }}$

IORT $=$ intraoperative radiotherapy, $\mathrm{pCR}=$ pathological complete response.

after definitive surgery defined as 'no tumour touching ink'. All patients re ceived adjuvant endocrine therapy; postmenopausal patients received an aromatase inhibitor, premenopausal patients younger than 40 received a $\mathrm{GnRH}$ analogue and tamoxifen and premenopausal patients older than 40 received tamoxifen. All patients received the same NACT regimen consisting of 4 cycles epirubicin and cyclophosphamide (EC) followed by 12 weeks of weekly paclitaxel.

\section{Statistical Analysis}

Follow-up data for each patient in the control group was censored to 396 days to ensure that the follow-up of the TARGIT and EBRT groups remained similar. This exclusion of additional 13 months of follow-up led to just 1 event being excluded from the EBRT group. Therefore, we believe that this methodology would not change the results of the analysis and at the same time counter the criticism of potentially unequal follow-up between the groups. Age and tumour size in $\mathrm{mm}$ were compared using the t-test. Categorical variables of grade, lymph node positivity and pathological complete response rate were compared using the Chi-square and Fisher's Exact test.

The following survival outcomes were analysed and compared between the TARGIT-Boost and EBRT-Boost groups: (1) overall mortality $(\mathrm{OM})$, event = any death, (2) breast cancer-specific mortality (BCSM), event = breast cancer death, (3) non-breast cancer mortality (NBCSM), event $=$ death from causes other than breast cancer, and (4) DFS, event = any relapse or death. For all outcomes patients were censored at the time of last follow-up. Kaplan-Meier curves were plotted and we estimated outcomes at the 5-year time point. All of the tests were 2 -sided, and a p value of $<0.05$ was regarded as statistically significant. We used the following software: the R system for statistical computing (version 3.0.1; R Development Core Team, Vienna, Austria, 2013), and STATA (version 14.0)

\section{Results}

Median follow-up was 49 months for both cohorts. No subject was lost to follow-up. Characteristics of the study population are shown in table 1. Age at the time of first diagnosis was the only significant difference between the 2 cohorts, with the TARGIT cohort being significantly younger than the EBRT cohort. The toxicity data for the cohort from which the experimental group receiv-
Table 2. Causes of death

\begin{tabular}{llll}
\hline Cause of death & IORT + & IORT - & Total \\
\hline Breast cancer & 0 & 1 & 1 \\
Myocardial infarction & 0 & 2 & 2 \\
Pulmonary embolism & 0 & 1 & 1 \\
Stroke & 0 & 1 & 1 \\
Total & 0 & 5 & 5 \\
\hline
\end{tabular}

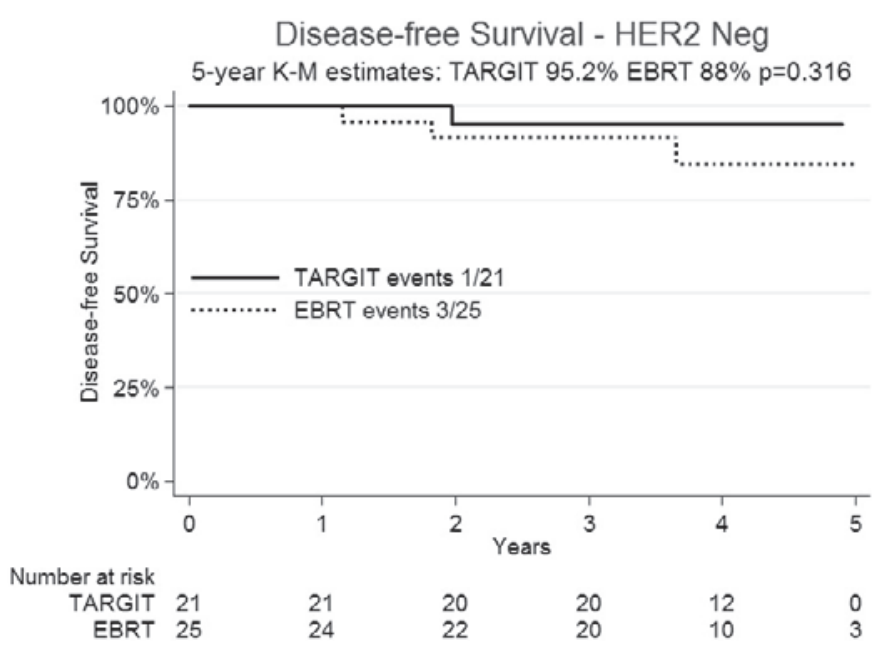

Fig. 1. Disease-free survival.

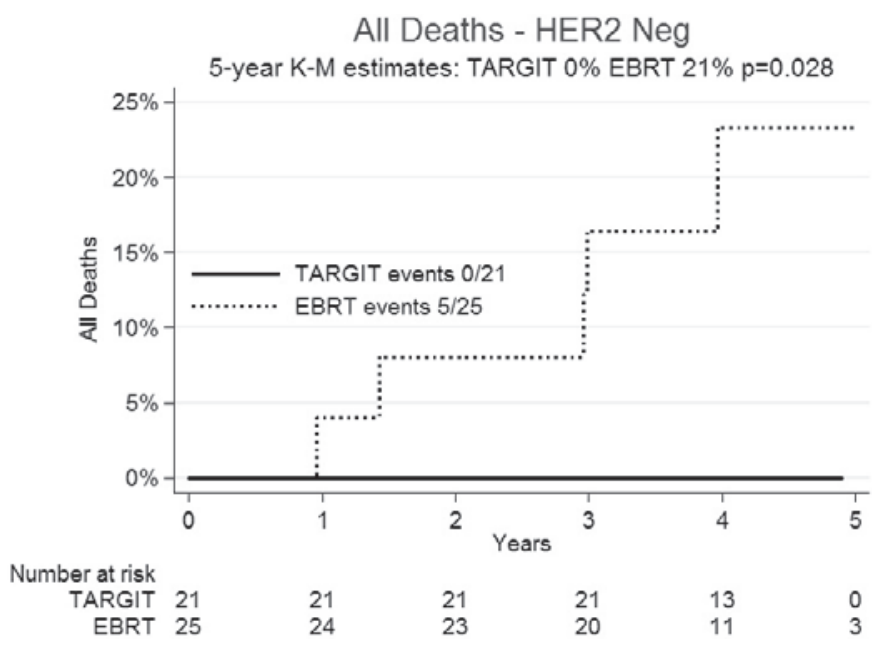

Fig. 2. Overall mortality.

ing TARGIT-IORT as a boost after NACT was extracted have been reported before and were comparable with the average postoperative morbidity after breast-conserving surgery in our institution.

There was no statistically significant difference between TARGIT and EBRT in terms of DFS and BCSM, although TARGIT fared numerically better than EBRT regarding these variables. OM was lower by $21 \%$ with TARGIT. This result and the difference in NBCSM of $16 \%$ were statistically significant. Causes of death are reported in table 2. Kaplan-Meier curves and $\mathrm{p}$ values can be found in figures $1-4$. 


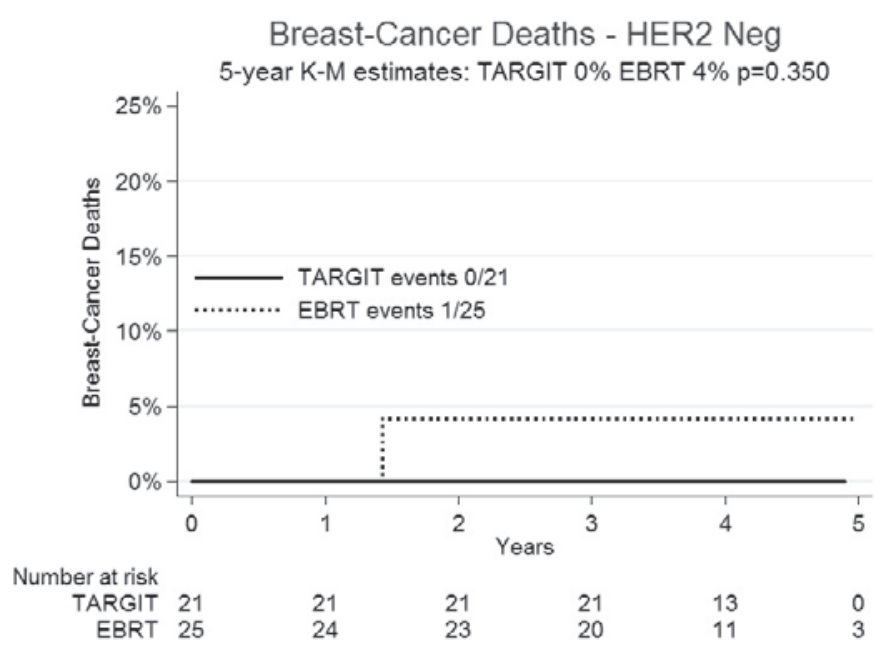

Fig. 3. Breast cancer-specific mortality.

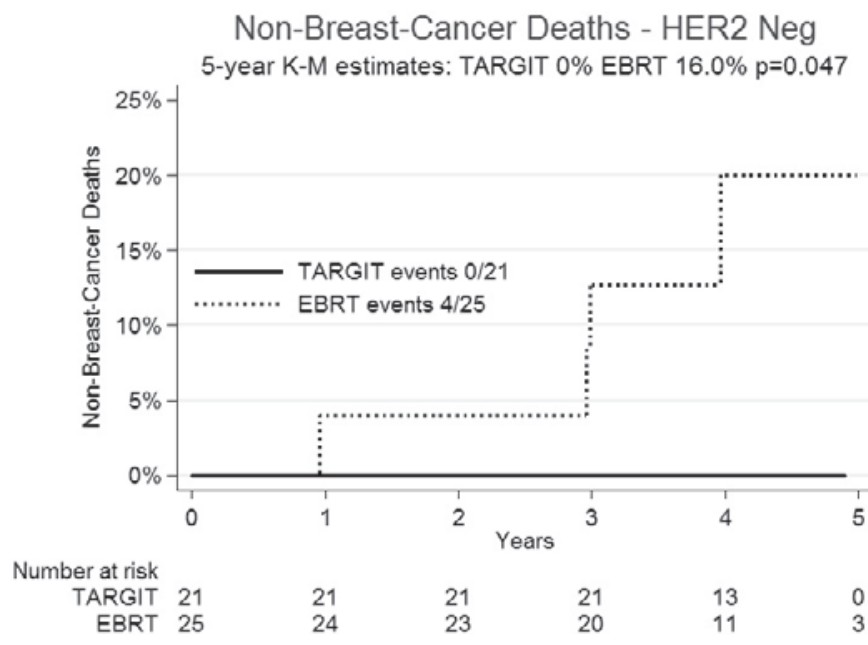

Fig. 4. Non-breast cancer-specific mortality.

\section{Discussion}

Initial reports about patients treated with TARGIT-IORT as an intraoperative boost during breast-conserving operations suggested that it might achieve superior local control [4, 5]. This approach has become a standard in several breast cancer centres, particularly in Germany and the United States. In the TARGIT A trial, in an attempt at radiation de-escalation, TARGIT-IORT was tested as the only radiotherapy in the course of breast-conserving therapy and was found not to be inferior to external whole breast irradiation in selected patients in a risk-adapted approach [2]. There has been concern regarding a possible higher rate of breast fibrosis found with TARGIT-IORT. An analysis of the TARGIT A population demonstrated that the rate of breast fibrosis was within the range seen with EBRT alone [15]. In previous studies of our group, we found that adapting this approach among patients who were undergoing breast-conserving therapy after neoadjuvant systemic therapy did not compromise cosmetic outcome [16], did not inter- fere with pathological evaluation of the margins and did not alter re-excision rates [17].

Patients who are candidates for NACT are generally at high risk of local and distant relapse and of death from breast cancer [18, 19]. A retrospective analysis using a different technique of intraoperative radiotherapy - intraoperative electron radiotherapy (IOERT) - compared 83 patients receiving IOERT after NACT with a group of 26 patients receiving conventional EBRT boost and found a trend for superiority for IOERT [10].

In the non-randomized retrospective cohort analysis presented here, we compared intraoperative tumour bed boost using a $50 \mathrm{kV}$ $\mathrm{X}$-ray device with an external boost among patients undergoing breast-conserving surgery after NACT including only patients with HR-positive HER2-negative tumours. All patients received external beam whole breast irradiation. The rationale for our investigation was based on our findings regarding the whole cohort. In this previous analysis, patients receiving their boost as TARGIT-IORT had a statistically significant better OS [12]. Based on the hypothesis that this difference might be attributed to the subset of patients with the worst prognosis at baseline, we had then decided to look at patients with TN and HER2-positive tumours specifically. In this analysis neither DFS nor OS differed significantly between patients receiving an external boost and patients receiving TARGIT-IORT, although trends favoured TARGIT-IORT [13]. These findings triggered the analysis presented here assuming the effect in the unselected cohort must have been driven by the HR-positive HER2negative subgroup.

The groups compared here were well balanced, with age being the only significant difference. Of course this difference has to be addressed regarding the significant difference in OS driven by NBCSM. Considering the median follow-up of 49 months, the mean age of 51 years in the TARGIT group and 60 years in the EBRT group will probably not have influenced the NBCSM difference significantly since the short time survival probability is 0.99769726 for a female aged 51 in Germany and 0.99471509 for the age of 60 respectively [20].

Both cohorts received the same chemotherapy schedules and achieved similar rates of pCR. Endocrine therapies according to menopausal status were the same for both groups. However, even though we found no significant difference in patient characteristics between the 2 cohorts, a selection bias cannot be excluded because this was not a randomised trial.

In the TARGIT-A study [2], a trend for superior OS with TARGIT-IORT compared with EBRT was observed. This was mainly attributable to reduced mortality from causes other than breast cancer. It has been suggested that the favourable effects of IORT on surgical wound fluid may result in wider systemic beneficial effects that may have contributed to the reduced mortality seen in the TARGIT-A trial. A non-randomised comparison of the patients in the TARGIT-A trial who received IORT + EBRT versus those who received EBRT found a statistically significant reduction in NBCSM. There were no deaths from non-breast cancer causes in the IORT+EBRT group compared with 24 in the EBRT group $(0 / 218$ vs. $24 / 892, \log$-rank $p=0.012)$ [21]. An explanation sug- 
gested by the authors for this phenomenon was a potential influence of immediate IORT on local tumour microenvironment and wound fluid that could get absorbed and cause beneficial systemic effects. Tumour cell line experiments have shown that the stimulating effect of wound fluid after lumpectomy on breast cancer cell proliferation, motility and invasiveness is abrogated if the patient receives IORT during the lumpectomy [22]. An analysis of the same study group demonstrated an induction of miR-223 in the peritumoral breast tissue, which resulted in a downregulation of the local expression of epidermal growth factor (EGF) and a decreased activation of epidermal growth factor receptor (EGFR) after IORT as a possible explanation for this effect [23]. It has also previously been discussed that IORT during lumpectomy may change the systemic course of not just breast cancer but also that of ischemic heart disease for the better [24]. These findings suggest an influence of IORT on factors with an impact on the vascular system. Although we are extremely cautious regarding the interpretation of our data, it is remarkable that in our analysis no patient in the IORT group died, whereas the deaths in the EBRT group all had a cause relating to the vascular system (stroke, myocardial infarction and pulmonary embolism).

The outcome data reported previously by our group [12], and which are reproduced in the subgroup analysis presented here, seem to support the hypothesis that the benefit of IORT may not be limited to a local effect by simply avoiding a geographic miss. The significantly better OS in the unselected cohort as well as in the HR-positive HER2-negative subgroup was driven by the significantly better NBCSM. As mentioned above, a similar effect was demonstrated in the TARGIT-A study in the whole study population as well as in the subgroup of patients receiving whole breast irradiation after IORT, i.e. receiving the same therapy as our study population [21].

Due to the retrospective character of our trial, caution in the interpretation of these data is necessary, but our findings still confirm that the use of TARGIT-IORT as a boost yields results that are not worse than external boost irradiation. Regarding the supposed beneficial effect of TARGIT-IORT on NBCSM more research is needed. We are planning a matched-pairs analysis of our dataset for the more than 700 cases treated at our institution with TARGIT-IORT as an intraoperative boost compared with cases treated with an external boost. The focus will be on comorbidities related to the vascular system at baseline and NBCSM and mortality, especially due to stroke, ischemic heart disease, pulmonary embolism, and the influence of tumour biology. The data collection for this study is ongoing.

The hypothesis of possible systemic beneficial effects of IORT will be prospectively tested in the randomised TARGIT-B trial comparing TARGIT-IORT boost to an external boost in women who are either younger than 45 or have a higher risk of local recurrence. This trial is including a stratification for the use of NACT and planned subgroup analyses regarding tumour biology. We encourage active participation in the TARGIT-B trial.

In conclusion, in a previous analysis, we demonstrated non-inferior and numerically superior OS attributable to a lower NBCSM for TARGIT-IORT during lumpectomy after NACT as a tumour bed boost compared to an external beam radiotherapy boost in an unselected population. In our current analysis we have shown that this difference is driven by the HR-positive HER2-negative subgroup, although we acknowledge that our results have to be interpreted with caution due to a possible selection bias, the comparatively small sample size and a rather short follow-up for a HR-positive cohort. These data give further support to the inclusion of such patients into the randomised TARGIT-B trial testing whether IORT boost is superior to EBRT boost and the analysis of subgroups based on tumour biology in that trial. Furthermore, our results warrant further investigation on the effects of TARGIT-IORT on non-breast cancer-specific endpoints.

\section{Disclosure Statement}

Dr. Kolberg reports personal fees from Carl Zeiss meditec, TEVA, Theraclion, Novartis, Amgen, Janssen, GSK, LIV Pharma and Genomic Health outside the submitted work. Dr. Loevey reports personal fees from Carl Zeiss meditec outside the submitted work. Dr. Akpolat-Basci, Dr. Stephanou and Dr. Untch have nothing to disclose. Dr. Fasching reports personal fees from Roche, TEVA, Genomic Health and Pfizer, grants and personal fees from Novartis and Amgen outside the submitted work. Dr. Hoffmann reports personal fees from Roche, Pfizer, MSD and Riemser Pharma outside the submitted work. Dr. Bulsara reports grants and personal fees from Carl Zeiss meditec outside the submitted work. Dr. Vaidya reports grants from Photoelectron corporation 19961999, personal fees from Carl Zeiss, non-financial support and other from Carl Zeiss during the conduct of the study; and personal fees from Carl Zeiss meditec, non-financial support and other from Carl Zeiss meditec outside the submitted work. Dr. Liedtke reports personal fees from Celgene, TEVA, Pierre Fabre, Novartis, Amgen, Eisai, GSK, Roche and Genomic Health outside the submitted work. There has been no funding from any outside source for the submitted work.

\section{References}

1 Sedlmayer F, Reitsamer R, Wenz F, et al.: Intraoperative radiotherapy (IORT) as boost in breast cancer. Radiat Oncol 2017;12:23.

2 Vaidya JS, Wenz F, Bulsara M, et al.: Risk-adapted targeted intraoperative radiotherapy versus whole-breast radiotherapy for breast cancer: 5-year results for local control and overall survival from the TARGIT-A randomised trial. Lancet 2014;383:603-613.
3 Vaidya JS, Bulsara M, Wenz F, et al.: Pride, prejudice, or science: Attitudes towards the results of the TARGIT-A Trial of targeted intraoperative radiation therapy for breast cancer. Int J Radiat Oncol Biol Phys 2015;92:491-497.

4 Vaidya JS, Baum M, Tobias JS, et al.: Long-term results of targeted intraoperative radiotherapy (TARGIT) boost during breast-conserving surgery. Int J Radiat Oncol Biol Phys 2011;81:1091-1097.
5 Wenz F, Welzel G, Blank E, et al.: Intraoperative radiotherapy as a boost during breast-conserving surgery using low-kilovoltage X-rays: the first 5 years of experience with a novel approach. Int J Radiat Oncol Biol Phys 2010;77:1309-1314.

6 Fastner G, Hauser-Kronberger C, Moder A, et al.: Survival and local control rates of triple-negative breast cancer patients treated with boost-IOERT during breast-conserving surgery. Strahlenther Onkol 2016; 192:1-7. 
7 Kaufmann M, von Minckwitz G, Mamounas EP, et al.: Recommendations from an international consensus conference on the current status and future of neoadjuvant systemic therapy in primary breast cancer. Ann Surg Oncol 2012;19:1508-1516.

8 Liedtke C, Hatzis C, Symmans WF, et al.: Genomic grade index is associated with response to chemotherapy in patients with breast cancer. J Clin Oncol 2009; 27:3185-3191.

9 Huober J, von Minckwitz G, Denkert C, et al.: Effect of neoadjuvant anthracycline-taxane-based chemotherapy in different biological breast cancer phenotypes: Overall results from the GeparTrio study. Breast Cancer Res Treat 2010;124:133-140.

10 Fastner G, Reitsamer R, Ziegler I, et al.: IOERT as anticipated tumor bed boost during breast-conserving surgery after neoadjuvant chemotherapy in locally advanced breast cancer - Results of a case series after 5-year follow-up. Int J Cancer 2015;136:1193-1201.

11 Kolberg HC, Loevey G, Akpolat-Basci L, et al.: Intraoperative radiotherapy as a boost after neoadjuvant chemotherapy: DFS after a median follow-up of 4 years. J Clin Oncol 2015;33(suppl): abstr e12050.

12 Kolberg HC, Lövey G, Akpolat-Basci L, et al.: Targeted intraoperative radiotherapy tumour bed boost during breast conserving surgery after neoadjuvant chemotherapy. Strahlenther Onkol 2016;193:62-69.
13 Kolberg HC, Loevey G, Akpolat-Basci L, et al.: Targeted intraoperative radiotherapy tumour bed boost during breast conserving surgery after neoadjuvant chemotherapy in HER2 positive and triple negative breast cancer. Rev Recent Clin Trials 2017;12:93-100.

14 Jwa E, Shin KH, Kim JY, et al.: Locoregional recurrence by tumor biology in breast cancer patients after preoperative chemotherapy and breast conservation treatment. Cancer Res Treat 2016;48:1363-1372.

15 Sperk E, Welzel G, Keller A, et al.: Late radiation toxicity after intraoperative radiotherapy (IORT) for breast cancer: results from the randomized phase III trial TARGIT A. Breast Cancer Res Treat 2012;135:253-260.

16 Kolberg HC, Stephanou M, Akpolat-Basci L, et al.: Intraoperative Radiotherapie mit dem Intrabeam ${ }^{\circledR}$ als vorgezogener Boost bei der brusterhaltenden Therapie des Mammakarzinoms - Erfahrungen nach den ersten 200 Fällen. Senologie 2013;10:A73.

17 Kolberg HC, Stephanou M, Akpolat-Basci L, et al.: Intraoperative Radiotherapie mit dem Intrabeam ${ }^{\circledR}$ als vorgezogener Boost nach neoadjuvanter Chemotherapie des Mammakarzinoms - Erfahrungen nach den ersten 130 Fällen. Senologie 2014;11:A67.

18 Loibl S, Volz C, Mau C, et al.: Response and prognosis after neoadjuvant chemotherapy in 1,051 patients with infiltrating lobular breast carcinoma. Breast Cancer Res Treat 2014;144:153-162.
19 Krug D, Lederer B, Debus J: Relationship of omission of adjuvant radiotherapy to outcomes of locoregional control and disease-free survival in patients with or without pCR after neoadjuvant chemotherapy for breast cancer: A meta-analysis on 3481 patients from the Gepar-trials. J Clin Oncol 2015;33(suppl):abstr 1008

20 www-genesis.destatis.de.

21 Vaidya JS, Bulsara M, Wenz F, et al.: The lower nonbreast cancer mortality with TARGIT in the TARGITA trial could be a systemic effect of TARGIT on tumor microenvironment. Int J Radiat Oncol Biol Phys 2013: 87:S240.

22 Belletti B, Vaidya JS, D`Andrea S, et al.: Targeted intraoperative radiotherapy impairs the stimulation of breast cancer cell proliferation and invasion caused by surgical wounding. Clin Cancer Res 2008;14:1325-1332.

23 Fabris L, Berton S, Citron F, et al.: Radiotherapy-induced miR-223 prevents relapse of breast cancer by targeting the EGF pathway. Oncogene 2016;35:4914-4926.

24 Vaidya JS, Bulsara M, Wenz F: Ischemic heart disease after breast cancer radiotherapy. N Engl J Med 2013; 368:2526-2527. 